\title{
De Berlin à la conquête du monde
}

L'irrésistible expansion du döner kebab

\section{From Berlin to the World Conquest}

\author{
The Irresistible Expansion of Döner-Kebab
}

Stéphane de Tapia

Résumé: Le döner-kebab est devenu la spécialité emblématique de la cuisine turque, aujourd'hui consommé dans le monde entier. En réalité, c'est une préparation apparue relativement récemment en Turquie et sous sa forme internationale avec l'immigration turque en Allemagne. Il est revendiqué par les Turcs, les Grecs ou les Libanais, mais reste étranger aux autres pays turcophones, sauf importation récente apportée par les immigrés turcs. Gastronomes et gourmets turcs sont souvent attristés de voir que cette préparation est devenue le symbole de la cuisine turque à l'étranger.

Mots-clés : champ migratoire, cuisine turque, döner-kebab, Europe, Turquie, vente à emporter

Abstract: Döner-kebab became the emblem of Turkish gastronomy. It is nowadays present in all the world. In fact, this culinary preparation is somewhat new in Turkey, and in its worldwide form of sandwich, it appears in Germany with the immigration of Turkish workers, and being offered by the Turks, the Greeks and the Lebanese cooks. However, it is not known by the other Turkic countries, except if imported by Turkish migrants. Turkish Gastronoms and Gourmets are often sad to see that this speciality became the symbol of Turkish cuisine in foreign countries.

Keywords: Döner-kebab, Europe, migratory field, take-away, Turkey, Turkish cooking 
Les brochures et le site officiel du ministère turc du Tourisme et de la Culture le répètent à l'envi dans toutes les langues utilisées à l'adresse du tourisme international : " la cuisine turque est la troisième du monde ", se rangeant cependant prudemment derrière les deux premières incontestées dans l'imaginaire ou les représentations, pas seulement du touriste international moyen, la française et la chinoise. Certes, la gastronomie turque est tout sauf mauvaise ou même moyenne : il existe en Turquie, dans les métropoles, d'excellents restaurants dirigés par d'excellents chefs, une grande variété de traditions culinaires régionales, dues sans aucun doute à une grande variété de terroirs et de produits, mais aussi aux origines d'une population issue d'un vaste empire. Mais comme le dit la sagesse populaire : " ah oui, mais à la maison, c'est autre chose !».

Et le döner kebab dans tout ça ? Invention vraiment turque? A moins qu'elle ne soit grecque (gyros) ou libanaise (shawarma) ? De fait, cette viande qui tourne sur elle-même, sur une broche verticale, grillant lentement au feu de bois, mais de plus en plus dans un four tournant au gaz, ou électrique, fabriquée en Allemagne, et distribuée par des semi-remorques allemands, néerlandais, ou même polonais (!) en paquetages de 10,25, $50 \mathrm{~kg}$, commandés via Internet pour une livraison le lendemain matin, a conquis les cinq continents. En bref, partout où des Turcs, Kurdes, Grecs, Arméniens, Iraniens (la liste n’est sans doute pas exhaustive) se sont installés. Les nuances sont locales : sauce blanche à la turque ou à l'australienne ? Au ketchup ou à la mayonnaise ? Ou, pire que tout, les deux à la fois ! Portion à la " parisienne » ou à la "strasbourgeoise ", viande halal ou viande haram ? Lauteur de cet article se rappelle avoir entendu à la foire de Strasbourg un restaurateur local crier, avec un fort accent alsacien : « kebab aux trois viandes », la troisième étant du... porc! Chose que l'on ne se permettrait sans doute plus aujourd'hui devant les protestations de la « communauté turque » admirative du grand Président Erdoğan.

Quelques années auparavant, au salon du Tourisme de Colmar cette fois, le stand de la Bulgarie offrait un dépliant intitulé : « La cuisine bulgare, cette inconnue ». Force était de constater que, comme en Grèce, les spécialités, du reste appétissantes, portaient presque toutes des noms turcs, à moins qu'ils n’aient été grecs, ou arabes du Machreq... la différence majeure étant, comme dans le kebab alsacien, l'usage de la viande de porc.

Döner signifie tout bêtement "tournant / qui tourne ». On remarquera que c'est bien le sens du grec gyros, mais aussi de l'arabe shawarma, si ce n’est que ce terme dérive d'un autre mot turc, çevirme signifiant " le fait de tourner ». En turc, le çevirme (qui peut aussi signifier « la traduction», autre façon de tourner) s'appliquerait plutôt à ce que le Maghreb appelle le méchoui, mouton entier tournant sur une broche, mais horizontalement cette fois. L'historien Jean-Louis Bacqué-Grammont (1977), dans un ouvrage de vulgarisation touristique, donne un panorama assez étoffé de la gastronomie turque. Pour autant, il ne s’attarde pas spécialement sur le döner, décrit comme suit : « quartiers de viande empilés sur une broche verticale tournant autour d'un 
étagement de braises ardentes; on en tranche de fines lamelles bien grillées à l'extérieur ", sauf à ajouter le kebab d'İskender, qui a fait la fortune de son inventeur à Bursa et proposé dans tout restaurant turc du monde.

Quant au kebab, terme d'origine arabe, il désigne des morceaux, de tailles très variables, ou des boulettes, de formes très variées, grillés, frits, cuits en sauce, entrant dans de nombreuses préparations, allant du şiş kebab (brochette, dite ailleurs souvent shashlyk - encore un mot dorigine turque) au saç kebab (petits cubes de viande cuits sur une tôle-saç_posée sur un foyer), en passant par le kebap d'Adana, d'Antep ou d'Urfa, viande hachée grillée sur brochette (se munir d'une carte : plus la ville est à l'est, plus le kebab est épicé) ou le tas $k e b a b$, une sorte de ragoût en sauce. Les recettes sont en réalité très variées. Il est possible de trouver du döner au veau et au poulet, les plus courants, mais aussi au bœuf, à l'agneau, au cabri et, chez les non-musulmans (grecs, bulgares, serbes...), au porc. On peut tout aussi bien préparer des brochettes ou des boulettes (köfte) de poisson ou de fruits de mer. Le döner kebab n'est donc qu'une forme parmi tant d'autres, mais sans doute la plus marquante par son appareillage.

Il nest peut-être pas inutile de revenir aux classiques turcs de la gastronomie. Un très grand classique de la cuisine turque, Necip Usta [Ertürk] (1987) ne cite pas le döner alors qu'il décrit pourtant des modes et " machines » de cuisson traditionnels au four et à la broche : tandır (cuisson sur la braise ou la cendre, dans un four en terre), çevirme (au charbon de bois, au gaz, électrique), kuyu (puit creusé dans la terre). Plus ancien, le livre de K. Adîl (1933) règle en sept lignes, page 196, la recette 339 : döner kebab (!), prouvant d'ailleurs au passage qu'on peut aussi le faire chez soi.

Pour qui s'intéresse aux cuisines du monde, grand voyageur ou simple curieux, parisien, berlinois ou new-yorkais, le fait est que la mondialisation et les migrations internationales mettent à portée de fourchette ou de baguette-c'est selon-gastronomies italienne, indienne, thaïlandaise, japonaise, coréenne, vietnamienne, mexicaine, libanaise, marocaine, arménienne, et bien d'autres encore, qui toutes pourraient revendiquer la «troisième place » après la française et la chinoise qui nont quà bien se tenir. Prétentions qui rappellent la guerre commerciale autour des vins français attaqués de toute part par de nouveaux produits moins chers et dont la qualité augmente avec le temps.

\section{Cuisine impériale, cuisines régionales}

Comme les cuisines chinoises ou indiennes, la cuisine turque peut remonter aux palais ottomans et en particulier au Palais impérial de Topkapı, encore impressionnant par ses cuisines aujourd'hui transformées en musée des porcelaines et ustensiles de cuisine. Les Sultans et leurs proches disposaient de cuisiniers nombreux, entourés de métiers spécialisés (bouchers, boulangers, 
pâtissiers...), et la gastronomie moderne s'en inspire encore largement. Cette gastronomie repose sur l'immensité d'un empire-entre frontières autrichiennes et iraniennes, des rives septentrionales de la mer Noire aux déserts du Sahara et d'Arabie, qui mixait populations, traditions et produits, au moins au Palais ; elle mêle encore sans doute origines nomades centrasiatiques et traditions byzantines, mais aussi « coloniales » (terme que récuserait évidemment l'historiographie turque) : albanaises, tatares, arabes, nord-caucasiennes... (Demirgül 2018; Sauner-Leroy 2000). Les régions, en l'absence de moyens de transports modernes, gardaient leurs originalités : huile d'olive contre graisses animales, beurre ou queue de mouton, poissons et légumes des côtes face aux céréales des hautes terres anatoliennes, conservation sédentaire et nomade des viandes des laitages et des fruits secs... Les dénominations turques, ou plutôt ottomanes, envahissent alors les langues hongroise, serbe, roumaine, grecque, arabe. Mais on oublie que beaucoup de termes sont déjà à l'origine arabes ou persans, voire plus rarement chinois. Les légumes et fruits ont des noms à consonances arabes et persanes-à la notable exception de l'ail et de l'oignon communs au turc et au mongol, de la pomme, commune au turc et au hongrois-, tout comme les épices ou les desserts, alors que les poissons et fruits de mer relèvent presqu'exclusivement du grec (sauf les espèces d'eaux douces).

Les influences réciproques sont souvent difficiles à déterminer. Existe-t-il une cuisine azerbaïdjanaise différente de la cuisine iranienne ou turque ? Estelle vraiment originale ou une symbiose / osmose entre les deux traditions? " Bien sûr que oui ! », répondront à coup sûr les Azerbaïdjanais, " mais non, mais non !", répondront les Turcs comme les Iraniens qui partagent kebab / kabab, polo / pilâv / plov / palau... avec les Ouzbeks ou les Ouïgours ou les Afghans ou les Indo-Pakistanais. D'où provient le mantı, raviole cuite au four ou à l'eau bouillante, mais simple pâte coupée en petits carrés à Kayseri, alors que la spécialité est présente de la France au Japon, en passant par l'Italie et la Russie, mais que le terme commun existe, lui, avec des variations, en turc de Turquie, du Caucase, d'Asie centrale, en chinois (wonton), coréen (mandu) et japonais (bunto), aux côtés de bien d’autres dénominations, selon le mode de cuisson, la pâte—blé ou riz—, la taille, la forme, la farce, la sauce etc. ?

L'historique précis de la spécialité est tout aussi difficile à tracer, car plusieurs théories sont en présence. Le döner kebap semble être apparu vers 1830 avec un cuisinier nommé Hamdi Usta dans la ville de Kastamonu (Anatolie nord-occidentale) sur une broche horizontale, puis, vers 1850, à Bursa avec İskender Efendi-dont les descendants développent l'entreprise-, sur une broche verticale, mais le photographe britannique James Robertson en prend des clichés à Istanbul dès 1854-1855, dont un coin de boutique ou restaurant. Plusieurs sources font un rapprochement avec une spécialité de la région d'Erzurum nommée că̆ kebap (sur broche tournante horizontale) ou Tortum kebap ou encore Oltu kebap, selon la petite ville où on la prépare. Mais entre ceux qui mettent en avant l'étymologie arménienne du terme et ceux qui veulent à tous prix établir la turcité de l'origine, la polémique peut encore 
naître (Serçeoğlu 2014, Üzümcü et Denk 2019). De fait, tous les textes « historiques »-y compris les media et toutes les versions de Wikipedia-reprennent ces éléments exposés par un enseignant et restaurateur (Renan Yaman), et repris par tous les « historiens » de la recette (Cebirbay et Aktaş 2015).

\section{Lémigration turque, vecteur de la gastronomie turque?}

Il existerait plus de 550 points de vente de kebab à Paris, 11000 dans toute la France. La Grande-Bretagne en compterait 17 000, ouverts par des Chypriotes grecs et turcs, la Finlande un millier. L'Allemagne, avec environ 16000 points de vente, en consommerait deux milliards d'unités par an, soit 400 tonnes par jour produites par 350 usines ou ateliers, pour un chiffre d'affaires de 2,5 à 3,5 milliards annuels en 2010-2011, 4,5 milliards aujourd'hui. Il existe d'ailleurs en Allemagne une Association européenne des producteurs turcs de Döner (ATDİD-Avrupa Türk Döner İmalatçıları Derneği / Verein Türkischer Dönerhersteller in Europa). La France, aux mêmes dates, en consommerait de 280 millions (2011) à 360 millions (2019) annuellement, et 78 pour cent des jeunes Français de 18 à 24 ans en auraient consommé. S’agit-il pour autant d'un ambassadeur de la gastronomie turque, voire d'une forme de soft-power? Le döner montrerait les capacités turques d'ubiquité, d'adaptation, de dynamisme économique, déchaînant parfois les passions (Cassely, Fourquet et Manternach 2019). Il est symptomatique que l'encyclopédie Wikipedia lui consacre douze pages en version anglaise, neuf en version allemande, mais seulement trois en version turque.

L’immigration turque est un phénomène récent (d’après les années 1950), mais quasi-mondial et de facto transnational. Les plus gros effectifs sont présents en Allemagne, avec signature d'un premier accord bilatéral en 1961, suivie par la France et les Pays-Bas. Ce que l'on sait moins, c'est que cette émigration s'est installée progressivement dans toute l'Europe, y compris en Europe orientale après la Perestroïka, dans les pays arabes producteurs d'hydrocarbures dès 1972, parallèlement en Amérique du nord et en Australie, aujourd'hui en Afrique du nord et sub-sahélienne. Sans réelle tradition migratoire, mais en réalité historiquement très mobile, la population musulmane turque (d’autres groupes ottomans comme les Syro-libanais dits los Turcos en Amérique latine ou les Arméniens avaient déjà ouvert la voie, et une version latina du döner, dite Al Pastor au Mexique et en Amérique du sud, terres d'installation de los Turcos, existe aussi) sest engouffrée dans les portes ouvertes, avec un solide esprit d'autonomie qui a surpris plus d'un chercheur spécialiste de l'Empire ottoman. Une encyclopédie azerbaïdjanaise de cuisine, fort intéressante au demeurant (Omiraslanov 2007), cite le döner dans l'article «kabab», mais sans autre précision qu'une "sorte de kebab célèbre dans le monde est présent en Azerbaïdjan », ce qui pourrait signifier qu'il a été apporté par l'immigration turque, très visible à Bakou pour qui sait décrypter les devantures des magasins 
et restaurants. Aucun ouvrage, aucune brochure, édités par le ministère azerbaïdjanais du Tourisme ne cite le döner, montrant bien qu'il s'agit là d'une importation récente. Même constat dans les guides ouzbeks ou kazakhstanais, la cuisine du Kazakhstan étant bien plus proche de celle de la Mongolie et de la tradition purement nomade, avec viandes bouillies plus que grillées ou rôties et forte présence de la viande chevaline, élément complètement incongru, sinon harâm, pour les Turcs (Kenjeaxmetuly 2010).

Il existe de très nombreux travaux aussi bien sur la construction du champ migratoire turc que sur la création d'entreprises par les migrants turcs. Nermin Abadan-Unat (2006), sociologue autant que politologue, a accompagné par ses très nombreux travaux cette folle aventure. Dès 1965, elle édite la première grande étude sérieuse sur l'immigration turque en Allemagne. En 2002, elle publie une grande "fresque " sur lémigration, plusieurs fois rééditée. Mais dès 1972, elle remarque dans la revue française Sociologie $d u$ Travail que les migrants n’auront aucune envie de rentrer au pays, préférant devenir indépendants, souvent créateurs de petites entreprises (Abadan 1972). Les restaurants, souvent des TPE (Très petite entreprise) plutôt que des PME (Petite et moyenne entreprise), se multiplient dès les années 1970. Le döner kebab devient le fer de lance de la "cuisine turque ", à tel point que le terme, très réducteur, de kebab s'impose aussi pour létablissement de restauration, quelle que soit sa taille. La forme européenne du döner, peu fréquente en Turquie, au grand étonnement des touristes, présente l'intérêt dêtre souple (on peut $\mathrm{y}$ ajouter ce que l'on veut), calorique, roborative, mais au total peu chère ; elle convient aux petits budgets (étudiants, ouvriers, employés, chauffeurs...) comme aux gens pressés. Elle entre facilement dans la catégorie fast-food et peut convenir aux food-trucks (surtout depuis la fabrication en usine, nul n'est besoin de cuisine), s'apparente aux hamburgers, tacos, sandwichs tunisiens et autres sandwichs en tous genres. L'investissement, dès lors que l'entreprise se concentre sur ce produit, n'est pas important : un tout petit local peut faire l'affaire.

Alors que les vrais restaurants turcs de qualité restent rares-contrairement aux restaurants libanais, voire iraniens, qui jouent bien plus la carte gastronomique - , le kebab devient un enjeu quasi-identitaire en matière d'intégration ou de ségrégation, subie ou revendiquée par les acteurs (Çağlar 1998). La droite identitaire hurle " au loup ", mettant en avant, comme pour les restaurateurs chinois, l'absence d'hygiène, l'emploi de travailleurs clandestins, l'opacité des intrants, la fraude fiscale et pourquoi pas la vitrine du terrorisme, selon le moment, du PKK kurde ou de l'islamisme radical. La gauche libérale y voit au contraire la preuve de l'intégration par la création d'entreprise et d'emplois, l'autonomie des acteurs économiques et sociaux, la vitalité du modèle d'intégration à la française (Jean-Laurent Cassely et al., 2019). 


\section{Le döner kebab, légende urbaine?}

Il existe de nombreuses façons de présenter au consommateur le döner kebab: sur une assiette ou un plat offrant des grillades variées (côtelettes d'agneaupirzola, boulettes-köfte, brochettes-şiş...), accompagné de yoğurt et de tomates ou sauce, à la façon du chef İskender de Bursa / İskender kebab, accompagné de salades et crudités, bulgur ou frites... Mais le döner « né en Allemagne » est présenté en sandwich ou dans un pain rond fendu en deux (pide / pita) ou dans une galette (yufka et dürüm), et même depuis peu sur un lahmacun, variante turque de la pizza, mais avec du pain sans levain, donc sur une couche... de viande hachée. En fait, tout est possible !

Le sandwich berlinois devenu mondial aurait été inventé par Mehmet Aygün, véritable entrepreneur à la tête, avec ses fils, d'une chaîne d'hôtels en Turquie... A moins que ce ne soit par Kadir Nurman, autre immigré turc établi à Berlin, mais ayant nettement moins la fibre commerciale, cependant distingué par ses pairs comme le véritable inventeur. A moins que ce ne soit un autre petit restaurateur, Nevzat Salim, de Reutlingen. La guerre du döner est donc ouverte entre immigrés turcs, mais aussi entre Turcs et Grecs et Libanais. Il semble qu'il n'y ait pas de sources scientifiques sur cette guerre entre inventeurs par médias interposés, mais c'est justement ce qui fait la légende urbaine. Les sites (culinaires, plus ou moins gastronomiques, d'information plus générale, pages loisirs, culture, etc.) reprennent souvent les mêmes informations, données, avis, commentaires..., chacun ayant fait la démarche d'écrire la "véritable histoire du döner kebab " avec son lot de représentations hasardeuses. Ainsi, l'image de cavaliers ottomans parcourant la steppe centre-anatolienne, épée garnie de viande au soleil (à un moment où les Ottomans nexistaient pas encore !) renvoie aux cavaliers d'Attila inventeurs du steak " cuit » sous la selle comme au steak «tartare » qui n'a de Tatar que le nom, sauf si l'on se rappelle que la tribu tatare des origines avait la réputation d’empoisonner ses hôtes (comme Yesugey, le père de Gengis Khan, qui s'est vengé en déclenchant ce qu'on qualifierait aujourd'hui de génocide). Les campements tatars seraient ainsi les ancêtres de "l’auberge espagnole ", chacun préférant, par précaution, apporter ses ingrédients.

Les étymologies proposées sont tout aussi « flottantes », sans être totalement fantaisistes. Tout le monde a compris que la racine DÖN signifie TOURNE, chacun déclinant la forme verbale turque selon ce qu'on lui aura dit. Il est intéressant de constater que la même racine peut nous donner " converti », ce qui est le cas des «Dönme », population juive d'Istanbul ayant accepté de se convertir à l'islam, mais aussi des Döngen / Dönen, population de culture chinoise devenue musulmane sunnite. Ces derniers s'auto-désignent comme Hui.

De fait, l'ATDİD, citée plus haut, créée à Berlin en 2006, rassemble de nombreux producteurs dont certains ont acquis une véritable envergure industrielle, avec plusieurs dizaines ou centaines d'employés, des flottes de camions frigorifiques-comme les producteurs de sucuk, salam et autres sosis halal 
(variétés de charcuterie turque, mais seul le sucuk ressort de la tradition) ou ceux de beyaz peynir, nom turc de la feta, terme maintenant déposé par la Grèce-et une envergure européenne. Un simple relevé des pages internet des entreprises produisant du döner industriel permet de citer Karmez, BirTat, Öztürk, Öztat, Öztaş, Polat, Aydın, Galileo, Kaplan, Fırat, Final-Fa, Döner Welt, Atik, Milâs, Hisar et tant d'autres, qui toutes disposent de leur site web, systèmes de commandes et livraisons. Certaines, comme Karmez, ont même réussi une implantation transnationale, en essaimant de l'Allemagne à l'Espagne, partant de l'idée que les nombreux touristes de la Costa Brava sont allemands, donc grands consommateurs de kebab alaturka. Les bulletins des associations patronales turques d'Allemagne vont jusqu'à mettre en scène une Angela Merkel, Chancelière de son état, en train de couper sur la broche un kebap, avec un grand sourire complice et gourmand.

Il existe cependant au moins une thèse récente sur la cuisine turque régionale : le géographe Pierre Raffard (2014) a travaillé sur les migrations internes en Turquie et sur l'image de la ville de Gaziantep, non loin de la frontière syrienne, qui ont investi sciemment la culture culinaire. Gaziantep s'enorgueillit de ses baklavas-inscrits par l'UE en 2013 aux Indications géographiques protégées (IGP)—, kadayıf et autres künefe, desserts à la croisée des traditions culinaires arabes, persanes et turques, mais aussi de 250 spécialités locales qui lui ont permis de figurer aussi sur la liste des cités créatives de l'UNESCO en 2015. Un entrepreneur régional, le pâtissier Güllüoğlu y a créé son entreprise en 1871, avant de s'installer en 1949 à Istanbul et en 2005 à New York et dans d’autres métropoles américaines et européennes. Là aussi, la guerre des « origines » règne car d’autres familles Güllüoğlu venues d'Antep déclarent être les premières, comme Karaköy Güllüoğlu, née en 1820 à Istanbul.

Le département voisin d'Urfa pourrait lui aussi mettre en avant ses traditions culinaires si l'on en croit une publication soutenue par les pouvoirs locaux (Durmaz 2014). Là aussi, riche tradition à la croisée des cuisines et produits méditerranéens, anatoliens et moyen-orientaux, où le döner est totalement absent des recettes de kebab. Le même constat peut être établi à Kayseri, au centre de l'Anatolie, qui s'enorgueillit aussi d'une riche tradition culinaire, ici également soulignée par la collectivité locale (Akkor 2019)

Le döner y est sans doute aujourd'hui bien présent comme dans la région du sud-est mais, comme partout ailleurs, en simple qualité de produit " immigré » récent. Ni à Gaziantep, ni à Şanlı Urfa, contrairement à Bursa, il n’est l'image qui prévaut. Car c'est à Bursa que le cuisinier nommé İskender (forme arabo-turque d'Alexandre, d'où " kebab d'Alexandre " dans de nombreux restaurants) a inventé le plat qui porte son nom à la fin du dix-neuvième siècle, agrémentant le döner de sauce tomate, yaourt, décoré de tomates et piments grillés, le tout nappé de beurre fondu. Ses descendants continuent la tradition. Lélévation générale du niveau de vie turc, la généralisation des transports modernes en Turquie et autour de la Turquie, la construction d'un immense champ migratoire créateur de milliers d'entreprises et une intense 
circulation migratoire entre Turquie et pays d'immigration auront fait le reste : le döner kebab, parce qu'il est peu cher, calorique et nourrissant, a conquis le monde.

\section{En guise de conclusion}

Il est donc tout à fait intéressant de constater que le döner kebab, universellement connu, s'il est bien né en Turquie il y a un ou deux siècles selon les sources, est bien moins présent en Turquie que dans le champ migratoire turc aujourd'hui étendu de l'Europe à l'Amérique et à l'Océanie. C'est une spécialité urbaine de l'Anatolie du nord-ouest et de la ville d'Istanbul, au départ très peu présente dans le reste du territoire turc. Elle s'y est imposée et généralisée avec la modernité dans la société urbanisée de la seconde moitié du vingtième siècle. En revanche, dans les villes européennes, puis mondiales, de résidence des immigrés turcs, le kebab est devenu le symbole d'une cuisine turque alors très peu connue par les sociétés d'accueil, principal ambassadeur de la gastronomie turque, au grand dam des cuisiniers turcs qui pensent valoir bien mieux que cela.

Stéphane de Tapia est géographe, professeur au Département d’études turques de la Faculté des langues, cultures, littératures étrangères et régionales de l'Université de Strasbourg, membre du laboratoire Dynamiques européennes (UMR6783). Il a été chercheur au CNRS entre 1990 et 2014, d'abord à Poitiers (MIGRINTER), puis à Strasbourg à partir de l'automne 1997, sur les questions de migrations et mobilités, transports et échanges. Il a publié en 2012 avec Marcel Bazin, La Turquie: Géographie d’un pays émergent, Paris, Armand Colin (Collection U) et en 2006, Migrations et diasporas turques : Circulation migratoire et continuité territoriale. Paris, Maisonneuve \& Larose et Istanbul, IFEA (Passé ottoman, présent turc).

Emails : stephane.detapia@misha.fr ; sdetapia@unistra.fr

Stéphane de Tapia is a geographer and a professor in the Department of Turkish Studies of Foreign and Regional Languages, Cultures, and Literatures of the Strasburg University. A Member of the European Dynamics Reserch Team (UMR6783), he was a social researcher first in Poitiers, then in Strasburg and worked about Migrations \& Mobilities, Transportation \& Exchanges. He published with Marcel Bazin in 2012: La Turquie: Géographie d'un pays émergent, Paris, Armand Colin (Collection U), and, in 2006: Migrations et diasporas turques: Circulation migratoire et continuité territoriale, Paris, Maisonneuve \& Larose + Istanbul, IFEA (Passé ottoman, présent turc). Emails : stephane.detapia@misha.fr; sdetapia@unistra.fr 


\section{Bibliographie}

Abadan, N. (1972), 'Le non-retour à l'industrie, trait dominant de la chaine migratoire turque', Sociologie du Travail 14, no. $3: 278-293$.

Abadan-Unat, N. [2002] (2006), Bitmeyen Göç. Konuk Isş̧ilikten Ulus-ötesi Yurttaşlı̆̆a [La migration qui n’en finit pas : Du travail invité à la citoyenneté transnationale] (Istanbul : Bilgi Üniversitesi).

Adîl, K. (1933), Aşçı ve Aşhane. Mükemmel Yemek Kitabı [Le cuisinier et la cuisine : Livre de repas extraordinaires] (Istanbul : Tefeyyüz Kitaphanesi-Ankara Maatbası).

Akkor, Y. E. (2019), Geçmişte Geleceğe Kayseri Mutfağı [Du passé à l’avenir, la cuisine de Kayseri] (Kayseri : Büyükşehir Belediyesi Kültür Yay).

Bacqué-Grammont, J.-L., photographies de R. et S. Michaud (1977), Voir la Turquie (Paris : Hachette Réalités), voir Glossaire et conseils gourmands, 124-125.

Cassely, J.-L., Fourquet, J., Manternach, S. (2019), 'Des dimensions politique, socioculturelle et territoriale des Kebabs en France', Fondation Jean Jaurès, https:// jean-jaures.org/nos-productions.

Caglar, A. (1998), 'McDoener: Doenerkebab und der Kampf der Deutsch-Tuerken um soziale Stellung' [McDoener: Le Döner Kebab et le combat des Turco-allemands pour un statut social], Sociologus. Zeitschrift fuer empirische Ethnosoziologie und Ethnopsychologie 48, no. 1: 17-41.

Caglar, A. (1998), 'Fast Food and Ethnic Business: The Story of Doener Kebap in Berlin', in Changing Food Habits: Case Studies From Africa, Latin America and Europe, (ed.) C. Lentz, (New York: Gordon and Breach).

Cebirbay, M. A. et Aktaş, N. (2015), 'Türk Mutfağı’nın Geleneksel Yiyeceği: Döner kebap, [Une spécialité traditionnelle de la cuisine turque : le Döner Kebab], www.ayk.gov.tr/up-contents/uploads/2015/01.

Demirgül, F. (2018), 'Çadırdan Saraya Türk Mutfağı' [Turkish Cuisine from the Tent to the Palace], Uluslararası Türk Dünyası Turizm Araştırmaları Dergisi 3, no. 1 : 105-125.

Le Correc, J. (2020), Kebab: salade, tomate, pognon ?, France 5, Doc. du Dimanche, Tony Comiti Productions (suivi le 15.03.2020).

Durmaz, Ö. (ed.) (2014), Dünyanın en eski mutfă̆ı: Şanlıurfa Yemek Kitabı [La plus ancienne cuisine du monde : livre des recettes de Şanlıurfa] (Şanlıurfa : Şurkav).

Omiraslanov, T. (ed.) (2007), Azarbaycan Kulinariyası Onsiklopediyası [Encyclopédie de la cuisine d'Azerbaïdjan], (Bakou: Şərq-Qərb).

Ertürk, N. Usta (1987), Türk Mutfak Sanatı [L'Art de la cuisine turque] (Istanbul : Remzi Kitabevi).

İçduygu, A. et Kirişçi, K., (eds.) (2009), Land of Diverse Migrations : Challenges of Emigration and Immigration in Turkey (Istanbul : Bilgi University Press).

Kenjeaxmetuly S. (2010), Қазақ̧тың дарқ̧ан дастарқ̧аны [The Kazakh national Cuisine] (Almaty: AlmatyKitap).

Raffard, P. (2014), Migrations intérieures et citadinités : le rôle de l'alimentation dans les stratégies des Gaziantepli à Istanbul, (Thèse de géographie, Université de Paris-Sorbonne).

Sancar, F. (1997 à 2006), La cuisine turque, brochure gratuite (pour celles qui sont en notre possession), éditée par le ministère du Tourisme et de la Culture.

Sauner-Leroy, M.-H. (2000), 'La cuisine ottomane ou la transmission d'un mode de vie', La Pensée de Midi 3, no. 3 : 36-46. 
Serçeoğlu, N. (2014), 'Yöre halkının mutfak kültürünü tanımı durumunun tespit edilmesi: Erzurum İli örneği' [Détermination de l'état de connaissance de la culture gastronomique populaire : L'exemple d'Erzurum], Journal of Tourism and Gastronomy Studies 2, no. $4: 36-46$.

Tapia, S. de, Soytürk, H. et Strigler, F. (2009), 'De la biographie des aliments à l'identité des adolescents', Les Cahiers de l'OCHA (Alimentations adolescentes en France), no. $14: 47-56$.

Tapia, S. de (2015), 'De la « cuisine nomade » à l'alimentation des émigrés : la Turquie, carrefour et creuset alimentaires', in Le Goût des autres : De l'expérience de l'altérité gastronomique à l'appropriation-Europe XVIIIe-XXIe siècles, (dir.) D. Saillard et D. Francfort, (Nancy : Presses universitaires de Nancy-Université de Lorraine).

Üzümcü, T. P., Denk, E. (2019), 'Erzurum ile özdeleşmiş bir lezzet : Oltu cağ kebabı' [Un goût représentatif d'Erzurum : le cag kebap d'Oltu], Journal of Tourism and Gastronomy Studies 7, no. $1: 463-483$.

Yaman, R. (1993), 'Döner kebabın Hikâyesi' [L'histoire du döner kebab], Türk Mutfak Kültürü Üzerine Araştırmalar, Türk Halk Kültürünü Araştırmalar ve Tanıtma Vakfı Yayınları, no. 3 : 92-101, http://www.renanyaman.de/htm/15.html. 